DOI: https://doi.org/10.15688/mpcm.jvolsu.2017.3.2

UDC 517

LBC 22.161

\title{
POINTWISE ESTIMATES OF SOLUTIONS AND EXISTENCE CRITERIA FOR SUBLINEAR ELLIPTIC EQUATIONS
}

\author{
Igor E. Verbitsky
}

Professor, Department of Mathematics,

University of Missouri

verbitskyi@missouri.edu

Columbia, MO 65211, USA

\begin{abstract}
We give a survey of recent results on positive solutions to sublinear elliptic equations of the type $-L u+V u^{q}=f$, where $L$ is an elliptic operator in divergence form, $0<q<1, f \geq 0$ and $V$ is a function that may change sign, in a domain $\Omega \subseteq \mathbb{R}^{n}$, or in a weighted Riemannian manifold, with a positive Green's function $G$. We discuss the existence, as well as global lower and upper pointwise estimates of classical and weak solutions $u$, and conditions that ensure $u \in L^{r}(\Omega)$ or $u \in W^{1, p}(\Omega)$.

Some of these results are applicable to homogeneous sublinear integral equations $u=G\left(u^{q} d \sigma\right)$ in $\Omega$, where $0<q<1$, and $\sigma=-V$ is a positive locally finite Borel measure in $\Omega$. Here $G(f d \sigma)(x)=\int_{\Omega} G(x, y), f(y) d \sigma(y)$ is an integral operator with positive (quasi) symmetric kernel $G$ on $\Omega \times \Omega$ which satisfies the weak maximum principle. This includes positive solutions, possibly singular, to sublinear equations involving the fractional Laplacian,
\end{abstract}

$$
(-\Delta)^{\frac{\alpha}{2}} u=\sigma u^{q}, \quad u \geq 0 \quad \text { in } \Omega,
$$

where $0<q<1,0<\alpha<n$ and $u=0$ in $\Omega^{c}$ and at infinity in domains $\Omega \subseteq \mathbb{R}^{n}$ with positive Green's function $G$.

Key words: sublinear elliptic equations, Green's function, weak maximum principle, fractional Laplacian.

\section{Introduction}

Let $\Omega$ be a connected open set in $\mathbb{R}^{n}$, or a weighted Riemannian manifold $(M, m)$ (see Sec. 2 below). We give a survey of recent results on positive solutions $u$ to the sublinear elliptic differential equation

$$
-L u+V(x) u^{q}=f, \quad x \in \Omega,
$$


where $0<q<1$, and

$$
L u=\operatorname{div}(\mathcal{A} \nabla u)
$$

is an elliptic operator with symmetric coefficients $\mathcal{A}=\left(a_{i j}(x)\right)_{i, j=1}^{n}$ in divergence form, $V$ and $f$ are functions in $\Omega$, and $f \geq 0$. Here $V(x)$ may change sign, and no extra boundary conditions are imposed on $V$.

One of the main goals is to obtain global pointwise estimates of $u$ in terms of the function $h$ which is defined as the minimal positive solution in $\Omega$ of the equation $-L h=f$, where $f \not \equiv 0$. We also consider positive solutions $u$ to the corresponding homogeneous equation

$$
-L u+V(x) u^{q}=0,
$$

with $f \equiv 0$. In Sec. 2, we present lower pointwise estimates of solutions to (1.1) and (1.3) obtained in $[16 ; 17]$.

There are also analogues of these estimates for positive (super) solutions $u$ to sublinear integral equations

$$
u+G\left(V u^{q}\right)=F,
$$

which involve the integral operator $G f(x)=\int_{\Omega} G(x, y) f(y) d y$ with a general positive kernel $G: \Omega \times \Omega \rightarrow(0,+\infty]$. If $G=G^{\Omega}$ is Green's function associated with the elliptic operator $L$ and $F=G f$, then (1.4) is in a sense equivalent to (1.1).

In many cases it is enough to assume that the kernel $G$ is lower semicontinuous, symmetric (or quasi-symmetric) and satisfies the weak maximum principle (see [16]), which includes many non-local operators, for instance, the fractional Laplacian $(-\Delta)^{\frac{\alpha}{2}}$. This leads to pointwise estimates of solutions for (1.4) in the case where $V=-\sigma \geq 0$, and $\sigma$ is a locally integrable function, or locally finite positive Borel measure in $\Omega$. In particular, we obtain sharp pointwise estimates of solutions (possibly singular) and existence results for the fractional Laplacian equation

$$
(-\Delta)^{\frac{\alpha}{2}} u=\sigma u^{q}, \quad u \geq 0 \quad \text { in } \Omega, \quad u=0 \quad \text { in } \Omega^{c},
$$

in general domains $\Omega \subset \mathbb{R}^{n}$ with positive Green's function $G$ for $0<\alpha \leq 2$, or nice domains (the entire space $\mathbb{R}^{n}$, or balls, or half-spaces in $\mathbb{R}^{n}$ ) for $0<\alpha<n$.

For entire solutions on $\Omega=\mathbb{R}^{n}$ and $V=-\sigma \geq 0$, where $\sigma$ is a locally finite positive Borel measure in $\mathbb{R}^{n}$, there are more complicated matching upper and lower bounds given in terms of certain nonlinear potentials of Th. Wolff type [8-10]. Earlier pointwise estimates for bounded positive solutions are due to Brezis and Kamin [6]. We consider such results in Sec. 3.

In the case $V=-\sigma \geq 0$, there are necessary and sufficient conditions for the existence of positive solutions $u \in \bar{L}^{q}(\Omega, \sigma)$ and $u \in L_{\mathrm{loc}}^{q}(\Omega, \sigma)$ to (1.4). The latter class includes all positive solutions $0<u<+\infty d \sigma$-a.e., and can be characterized either using certain localization techniques developed in [8] in the case $L=(-\Delta)^{\frac{\alpha}{2}}$ and $\Omega=\mathbb{R}^{n}$, or integral inequalities with weights as in [23] in the general case. These results are discussed in Sec. 3 and Sec. 4.

We also present in Sec. 5 criteria for the existence of positive solutions to (1.4) of higher integrability $u \in L^{q+\gamma}(\Omega, \sigma)(\gamma>0)$, which are easier to characterize and are useful in applications (see [27]). In particular, the case $\gamma=1$, i.e., solutions $u \in L^{1+q}(\Omega, \sigma)$, corresponds to finite energy solutions to sublinear elliptic equations (1.3) in the Dirichlet space $u \in W^{1,2}(\Omega)$ for uniformly elliptic operators $L$ in divergence form. 
Similar criteria for $\gamma>0$ can be applied to deduce conditions on $\sigma$ which ensure the existence of positive solutions $u$ to (1.3) in Sobolev spaces $W^{1, p}(\Omega)$ and Lebesgue spaces $L^{r}(\Omega, d x)$ for general domains $\Omega \subseteq \mathbb{R}^{n}$ with positive Green's functions (see [24;25]). Some earlier existence results of this type for $\sigma \in L^{s}(\Omega, d x)$ in bounded domains $\Omega$ are due to Boccardo and Orsina [4;5]. Such existence theorems under more general assumptions on $\sigma$ which include measures (possibly singular with respect to Lebesgue measure) are discussed in Sec. 6.

\section{Lower pointwise estimates of supersolutions}

In this section, we assume that $\Omega \subseteq \mathbb{R}^{n}$ is a connected open set, and consider the divergence form operator

$$
L u=\operatorname{div}(\mathcal{A} \nabla u)(x)=\sum_{i, j=1}^{n} \partial_{x_{i}}\left(a_{i j}(x) \partial_{x_{j}} u\right)
$$

where $a_{i j}$ are $C^{2}$ functions in $\Omega$, and $a_{i j}=a_{j i}$. Assume that $L$ is elliptic, that is, the matrix $\mathcal{A}=\left(a_{i j}(x)\right)$ is positive definite for any $x$ (the uniform ellipticity is not required).

Let us assume that the Dirichlet Green function of $L$ in $\Omega$ exists. We denote it by $G^{\Omega}(x, y)$. We set

$$
h(x)=G f(x)=\int_{\Omega} G^{\Omega}(x, y) f(y) d y,
$$

where $f \geq 0(f \not \equiv 0)$, and assume that $h(x)<\infty$ for all $x \in \Omega$ (note also that $h(x)>0$ in $\Omega$ ), and that the integral

$$
G\left(h^{q} V\right)(x)=\int_{\Omega} G^{\Omega}(x, y) h^{q}(y) V(y) d y
$$

is well-defined.

An interesting special case is when $f \equiv 1$, and $h$ is the solution of the Dirichlet problem:

$$
\left\{\begin{array}{l}
-L h=1 \quad \text { in } \Omega \\
h=0 \text { on } \partial \Omega .
\end{array}\right.
$$

In other words, $h(x)=\mathbb{E}_{x}\left[\tau_{\Omega}\right]$, where $\tau_{\Omega}=\inf \left\{t: X_{t} \notin \Omega\right\}$ is the first exit time from $\Omega$ of the (rescaled) Brownian motion $X_{t}$, and $x \in \Omega$ is a starting point. For bounded $C^{1,1}$ domains $\Omega$, it is well known that $h(x) \simeq d_{\Omega}(x)$, where

$$
d_{\Omega}(x)=\operatorname{dist}(x, \partial \Omega) .
$$

One of the main results of [17] in the case $0<q<1$ is the following global lower estimate for a (super) solution $u>0$ to (1.1).

Theorem 2.1. Let $\Omega \subseteq \mathbb{R}^{n}$ be a domain with a positive Green function $G^{\Omega}$. Suppose $V, f \in C(\Omega)$, and $f \geq 0, f \not \equiv 0$ in $\Omega$. Let $u \in C^{2}(\Omega)$ satisfy the inequality

$$
-L u+V u^{q} \geq f, \quad u>0 \text { in } \Omega .
$$

20 I.E. Verbitsky. Pointwise estimates of solutions and existence criteria for sublinear elliptic equations 
Set $h=G f$ and assume that $h<\infty$ in $\Omega$. Assume also that $G^{\Omega}\left(h^{q} V\right)(x)$ is well-defined for all $x \in \Omega$. Then the following estimate holds for all $x \in \Omega$ :

$$
u(x) \geq h(x)\left[1-(1-q) \frac{G\left(h^{q} V\right)(x)}{h(x)}\right]_{+}^{\frac{1}{1-q}} .
$$

This theorem is actually proved in [17] in a more general context of elliptic operators on weighted manifolds $(M, m)$. We recall that if $M$ is a smooth Riemannian manifold with the Riemannian metric tensor $g=\left(g_{i j}\right)$, then the associated Laplace - Beltrami operator $\mathcal{L}_{0}$ acts on $C^{2}$ functions $u$ on $M$ and is given in any chart $x_{1}, \ldots, x_{n}$ by

$$
\mathcal{L}_{0} u=\frac{1}{\sqrt{\operatorname{det} g}} \sum_{i, j=1}^{n} \partial_{x_{i}}\left(\sqrt{\operatorname{det} g} g^{i j} \partial_{x_{j}} u\right)
$$

Here $\operatorname{det} g$ is the determinant of the matrix $g=\left(g_{i j}\right)$, and $\left(g^{i j}\right)$ is the inverse matrix of $\left(g_{i j}\right)$. The Riemannian measure $m_{0}$ is given in the same chart by

$$
d m_{0}=\sqrt{\operatorname{det} g} d x_{1} \ldots d x_{n}
$$

so that $\mathcal{L}_{0}$ is symmetric with respect to $m_{0}$. Using the gradient operator $\nabla$ defined by

$$
(\nabla u)^{i}=\sum_{j=1}^{n} g^{i j} \partial_{x_{j}} u
$$

and the divergence div on vector fields $F^{i}$

$$
\operatorname{div} F=\frac{1}{\sqrt{\operatorname{det} g}} \sum_{i=1}^{n} \partial_{x_{i}}\left(\sqrt{\operatorname{det} g} F^{i}\right)
$$

we have

$$
\mathcal{L}_{0}=\operatorname{div} \circ \nabla
$$

Now let $\omega$ be a smooth positive function on $M$ and consider the measure $m$ on $M$ given by

$$
d m=\omega d m_{0} .
$$

The couple $(M, m)$ is called a weighted manifold, and $\omega$ in this context is called a weight (see [14]). The following operator $\mathcal{L}$

$$
\mathcal{L} u:=\frac{1}{\omega} \operatorname{div}(\omega \nabla u)=\frac{1}{\omega \sqrt{\operatorname{det} g}} \sum_{i, j=1}^{n} \partial_{x_{i}}\left(\omega \sqrt{\operatorname{det} g} g^{i j} \partial_{x_{j}} u\right)
$$

acting on $C^{2}$ functions $u$ on $M$, is called the (weighted) Laplace operator of $(M, m)$. This operator $\mathcal{L}$, which is symmetric with respect to measure $m$, can be used in place of the operator $L$ in $\Omega \subseteq \mathbb{R}^{n}$ above.

There is also a similar lower estimate for positive (super) solutions to the homogeneous equation (1.3). 
Theorem 2.2. Let $\Omega \subseteq \mathbb{R}^{n}$ be a domain with a positive Green function $G^{\Omega}$. Suppose $V \in C(\Omega)$, and $u \in C^{2}(\Omega)$ satisfies the inequality

$$
-L u+V u^{q} \geq 0, \quad u \geq 0 \text { in } \Omega,
$$

where $0<q<1$. Assume also that $G V(x)$ is well-defined for all $x \in \Omega$.

Then the following estimate holds for all $x \in \Omega$ :

$$
u(x) \geq[-(1-q) G V(x)]_{+}^{\frac{1}{1-q}} .
$$

Similar global pointwise estimates are obtained in [17] for all exponents $q \in \mathbb{R}(q \neq 0)$, but we do not consider the case $q \notin(0,1)$ here. Also, the preceding estimate remains true for nonnegative solutions $u$ if the integration in the Green's potentials $G^{\Omega}\left(h^{q} V\right)$ and $G^{\Omega} V$ respectively is taken over the set $\Omega^{+}=\{x \in \Omega: u>0\}$ in place of $\Omega$. Note that the differential inequalities (2.5) and (2.8) in Theorem 2.1 and Theorem 2.2 respectively are understood in the classical sense.

In the case $V=-\sigma \leq 0$, where $\sigma$ is a locally finite measure in $\Omega$, the preceding estimate yields

$$
u(x) \geq\left[(1-q) \int_{\Omega} G^{\Omega}(x, y) d \sigma(y)\right]^{\frac{1}{1-q}},
$$

which holds for all non-trivial weak solutions $u$ (possibly singular).

This estimate was obtained for the Laplacian $L=\Delta$ in the case $\Omega=\mathbb{R}^{n}$ in [6], without specifying the sharp constant $(1-q)^{\frac{1}{1-q}}$, under some additional assumptions on $\sigma \geq 0$ (see also [9]).

There are analogues of this estimate for integral operators with general positive kernels $G: \Omega \times \Omega \rightarrow(0,+\infty]$ on a locally compact Hausdorff space $\Omega$ that are lower semicontinuous, symmetric, and satisfy the weak maximum principle with constant $\mathfrak{b} \geq 1$ (see [16]).

For a locally finite Radon measure $v \in \mathcal{M}^{+}(\Omega)$, we denote by $G v$ the potential

$$
G \vee(x)=\int_{\Omega} G(x, y) d v(y) .
$$

We recall that the kernel $G$ is said to satisfy the weak maximum principle (with constant $\mathfrak{b} \geq 1$ ) provided that

$$
G \vee(x) \leq M, \quad \forall x \in \operatorname{supp} v \Longrightarrow G \vee(x) \leq \mathfrak{b} M, \quad \forall x \in \Omega,
$$

for any constant $M>0$ and $v \in \mathcal{M}^{+}(\Omega)$. When $\mathfrak{b}=1$, we say that $G$ satisfies the strong maximum principle.

These assumptions hold for many non-local operators $L$ of interest in nonlinear analysis, in particular, the fractional Laplacian operators $(-\Delta)^{\frac{\alpha}{2}}$ in domains $\Omega \subseteq \mathbb{R}^{n}$. For such kernels $G$ and $\sigma \in \mathcal{M}^{+}(\Omega)$, let

$$
G(f d \sigma)(x)=\int_{\Omega} G(x, y) f(y) d \sigma(y), \quad x \in \Omega,
$$

where $f \geq 0$ is a measurable function.

If $u>0 d \sigma$-a.e. is a supersolution, i.e.,

$$
G\left(u^{q} d \sigma\right)(x) \leq u(x)<\infty \quad d \sigma-\text { a.e. },
$$


then

$$
u(x) \geq(1-q)^{\frac{1}{1-q}} \mathfrak{b}^{-\frac{q}{1-q}}[G \sigma(x)]^{\frac{1}{1-q}} \quad d \sigma-\text { a.e. in } \Omega .
$$

The constant $(1-q)^{\frac{1}{1-q}}$ in $(2.13)$ in the case $\mathfrak{b}=1$ coincides with that in (2.10). Similar results hold for quasi-symmetric kernels $G$ such that there exists a constant $\mathfrak{a}>0$ for which

$$
G(x, y) \leq \mathfrak{a} G(x, y), \quad x, y \in \Omega,
$$

with a constant on the right-hand side of (2.13) depending on both $\mathfrak{a}$ and $\mathfrak{b}$. See [2] where quasi-symmetry is established for many elliptic operators in non-divergence form. Properties (2.11) and (2.14) will be used extensively in Sections 4 and 6 below.

\section{Matching upper and lower pointwise estimates of solutions}

In this section, we discuss recent results established in [8] for entire solutions to quasilinear elliptic equations of the type

$$
\begin{cases}-\Delta_{p} u=\sigma u^{q} & \text { in } \mathbb{R}^{n}, \\ \liminf _{x \rightarrow \infty} u(x)=0, & u>0,\end{cases}
$$

where $0<q<p-1$. Here $\Delta_{p}=\operatorname{div}\left(\nabla u|\nabla u|^{p-2}\right)$ is the $p$-Laplace operator, and $\sigma \geq 0$ is an arbitrary locally integrable function, or locally finite Borel measure, $\sigma \in \mathcal{M}^{+}\left(\mathbb{R}^{n}\right)$; if $\sigma \in L_{\text {loc }}^{1}\left(\mathbb{R}^{n}\right)$ we write $d \sigma=\sigma d x$.

The main goal is to give necessary and sufficient conditions on $\sigma$ for the existence of weak solutions to (3.1), and obtain matching upper and lower global pointwise bounds for positive solutions. In this approach, key integral inequalities are identified, and new nonlinear potentials of Wolff type are constructed that are intrinsic to a number of similar problems.

This approach is applicable to general quasilinear $\mathcal{A}$-Laplace operators of divergence type $\operatorname{div} \mathcal{A}(x, \nabla u)$ under standard boundedness and structural assumptions on $\mathcal{A}$, as well as to fully nonlinear $k$-Hessian operators, and the fractional Laplacian equations (see [8]).

We will restrict ourselves here to the case $p=2$ and linear operators $\operatorname{div}(\mathcal{A} \nabla u)$ under the uniform ellipticity assumption on the matrix $\mathcal{A} \in L^{\infty}\left(\mathbb{R}^{n}\right)^{n \times n}$. In this setup, the fundamental solution of the $\mathcal{A}$-Laplacian is known to be pointwise equivalent to that of the Laplacian, which reduces the problem to estimates involving Newtonian potentials.

In fact, we will consider below the fractional Laplacian equations,

$$
\begin{cases}(-\Delta)^{\frac{\alpha}{2}} u=\sigma u^{q} & \text { in } \mathbb{R}^{n}, \\ \liminf _{x \rightarrow \infty} u(x)=0, & u>0,\end{cases}
$$

for $0<q<1$ and $0<\alpha<n$; this includes the range $\alpha>2$ where the usual maximum principle is not available.

In the classical case $p=2$, equation (3.1), or equivalently (3.2) with $\alpha=2$, provides a model sublinear elliptic problem if $0<q<1$. It is easy to see that it is equivalent to the integral equation $u=N\left(u^{q} d \sigma\right)$, where $N \omega=(-\Delta)^{-1} \omega$ is the Newtonian potential of $d \omega=u^{q} d \sigma$ on $\mathbb{R}^{n}$. 
As we emphasize in [8], such problems are governed by the important integral inequality

$$
\left(\int_{\mathbb{R}^{n}}|\varphi|^{q} d \sigma\right)^{\frac{1}{q}} \leq \varkappa\|\Delta \varphi\|_{L^{1}\left(\mathbb{R}^{n}\right)},
$$

for all test functions $\varphi \in C^{2}\left(\mathbb{R}^{n}\right)$ vanishing at infinity such that $-\Delta \varphi \geq 0$. This inequality turns out to be necessary and sufficient for the existence of a positive solution $u \in L^{q}\left(\mathbb{R}^{n}, \sigma\right)$ to (3.1) if $p=2$ and $0<q<1$.

Inequality (3.3) represents the end-point case of the well-studied $\left(L^{p}, L^{q}\right)$ trace inequalities for $p>1$. A comprehensive treatment of trace inequalities in that case in terms of Sobolev capacities can be found in [22].

To characterize the existence of arbitrary weak solutions $u \in L_{\mathrm{loc}}^{q}\left(\mathbb{R}^{n}, \sigma\right)$, and obtain sharp global pointwise estimates, a localization procedure is used in [8] which involves the so-called intrinsic Wolff potentials.

More precisely, one needs to consider a localized version of (3.3) where the measure $\sigma$ is replaced with $\sigma_{B}=\chi_{B} \sigma$ restricted to a ball $B=B(x, r)$, and the corresponding best constant $\varkappa$ is denoted by $\varkappa(B)$. These constants are used as building blocks in the key tool, a potential of Th. Wolff type (see [1] for the theory and history of Wolff's potentials),

$$
K \sigma(x)=\int_{0}^{\infty} \frac{[\varkappa(B(x, r))]^{\frac{q}{1-q}}}{r^{n-2}} \frac{d r}{r}, \quad x \in \mathbb{R}^{n} .
$$

This nonlinear potential, together with the usual Newtonian potential $N \sigma$, provides sharp bilateral estimates of solutions in the case $p=2$ and $0<q<1$.

This work has been motivated by the results of Brezis and Kamin [6] who proved that (3.1), with $p=2$ and $0<q<1$, has a bounded solution $u$ on $\mathbb{R}^{n}$ if and only if No $\in L^{\infty}\left(\mathbb{R}^{n}\right)$; moreover, such a solution is unique, and there exists a constant $c>0$ so that

$$
c^{-1}(N \sigma(x))^{\frac{1}{1-q}} \leq u(x) \leq c N \sigma(x), \quad x \in \mathbb{R}^{n} .
$$

The lower bound in (3.5), which is generalized in (2.10) and (2.13), holds for all solutions. On the other hand, the upper bound is valid only for bounded solutions. Some generalizations of the upper bound to possibly singular solutions in the form

$$
u(x) \leq c\left[N \sigma(x)+(N \sigma(x))^{\frac{1}{1-q}}\right], \quad x \in \mathbb{R}^{n},
$$

under additional assumptions on $\sigma$, can be found in [9].

As was pointed out in [6], both the lower and upper estimates in (3.5) are sharp in a sense. However, there is a substantial gap between them. In Theorem 3.1 below this gap is bridged by using $K \sigma+(N \sigma)^{\frac{1}{1-q}}$. The corresponding pointwise estimates hold for all, possibly singular solutions, as well as more general nonlinear equations.

In the context of fractional Laplacian equations (3.2), we denote by $I_{\alpha}$ the Riesz potential of order $\alpha \in(0, n)$ on $\mathbb{R}^{n}$ :

$$
I_{\alpha} \mu=|x|^{\alpha-n} \star \mu=c(\alpha, n)(-\Delta)^{-\frac{\alpha}{2}} \mu,
$$

for $\mu \in \mathcal{M}^{+}\left(\mathbb{R}^{n}\right)$. In what follows, the normalization constant $c(\alpha, n)$ will be dropped for the sake of convenience; in particular, $I_{2} \mu=N \mu$ stands for the Newtonian potential. 
We now introduce some elements of nonlinear potential theory which is intrinsic to (3.2). Let $B=B(x, r)=\left\{y \in \mathbb{R}^{n}:|x-y|<r\right\}$ be a ball in $\mathbb{R}^{n}$, and let $\sigma_{B}=\chi_{B} \sigma$ stand for the measure $\sigma$ restricted to $B$. By $\kappa(B)$ we denote the least constant in the localized integral inequality

$$
\left\|I_{\alpha} v\right\|_{L^{q}\left(d \sigma_{B}\right)} \leq \kappa(B) v\left(\mathbb{R}^{n}\right), \quad \forall v \in \mathcal{M}^{+}\left(\mathbb{R}^{n}\right),
$$

where $0<q<1$ and $0<\alpha<n$.

We observe that the constant $\kappa(B)$ does not change if we restrict ourselves to absolutely continuous $v \in L_{+}^{1}\left(\mathbb{R}^{n}\right)$ on the right-hand side of (3.6).

We define the corresponding nonlinear potential of Wolff type by

$$
K_{\alpha, q} \sigma(x)=\int_{0}^{\infty} \frac{[\kappa(B(x, r))]^{\frac{q}{1-q}}}{r^{n-\alpha}} \frac{d r}{r}, \quad x \in \mathbb{R}^{n} .
$$

The following condition ensures that both $K_{\alpha, q} \sigma$ and $I_{\alpha} \sigma$ are not identically infinite:

$$
\int_{1}^{\infty} \frac{[\kappa(B(0, r))]^{\frac{q}{1-q}}}{r^{n-\alpha}} \frac{d r}{r}+\int_{1}^{\infty} \frac{\sigma(B(0, r))}{r^{n-\alpha}} \frac{d r}{r}<\infty,
$$

Clearly, the first part of (3.8) is equivalent to $K_{\alpha, q} \sigma \not \equiv+\infty$, whereas the second one is equivalent to $I_{\alpha} \sigma \not \equiv+\infty$ (and $I_{\alpha} \sigma<+\infty$ a.e.)

Note that solutions $u \in L_{\text {loc }}^{q}\left(\mathbb{R}^{n}, d \sigma\right)$ to (3.2) are understood in the weak sense.

Theorem 3.1. Let $0<q<1,0<\alpha<n$, and $\sigma \in \mathcal{M}^{+}\left(\mathbb{R}^{n}\right)$.

(i) If (3.8) holds, then there exists a minimal solution $u>0$ to (3.2) such that

$$
c^{-1}\left[K_{\alpha, q} \sigma+\left(I_{\alpha} \sigma\right)^{\frac{1}{1-q}}\right] \leq u \leq c\left[K_{\alpha, q} \sigma+\left(I_{\alpha} \sigma\right)^{\frac{1}{1-q}}\right],
$$

where $c>0$ is a constant which depends only on $q, \alpha$, and $n$.

(ii) Conversely, if there exists a nontrivial (super) solution $v$ to (3.2), then (3.8) holds, and $v \geq u$, where $u$ is the minimal solution of statement (i).

We observe that neither of the two parts of the condition (3.8) implies the other one. Condition $I_{\alpha} \sigma<\infty$ a.e. alone is not enough to ensure the existence of a global solution $u$ even if all the local embedding constants $\kappa(B)$ in (3.6) are finite, unless $\sigma$ is radially symmetric (see [9]).

\section{Existence of solutions for sublinear integral equations}

In this section, $G: \Omega \times \Omega \rightarrow(0,+\infty]$ is a lower semicontinuous, quasi-symmetric kernel on $\Omega \times \Omega$ that satisfies (2.14) along with the weak maximum principle (2.11), on a locally compact Hausdorff space $\Omega$. Let us fix a locally finite Radon measure $\sigma \in \mathcal{M}^{+}(\Omega)$.

In $[23 ; 25 ; 27]$, necessary and sufficient conditions are obtained for the existence of positive (super) solutions to the sublinear integral equation

$$
u=G\left(u^{q} d \sigma\right), \quad 0<u<+\infty \quad d \sigma-\text { a.e. in } \Omega,
$$

The existence of solutions in Lebesgue spaces, $u \in L^{\gamma}(\Omega, \sigma)$, turned out to be related to weighted norm inequalities of the type

$$
\|G(f d \sigma)\|_{L^{r}(\Omega, d \sigma)} \leq C\|f\|_{L^{s}(\Omega, d \sigma)}, \quad \forall f \in L^{s}(\Omega, d \sigma),
$$


for integral operators $G$ in the case $0<r<s<+\infty, s \geq 1$.

The most important and most difficult case is when $r=q(0<r<1)$ and $s=1$, i.e.,

$$
\|G(f d \sigma)\|_{L^{q}(\Omega, d \sigma)} \leq C\|f\|_{L^{1}(\Omega, d \sigma)}, \quad \forall f \in L^{1}(\Omega, d \sigma) .
$$

In the special case of Green's kernels and the corresponding elliptic operators $L,(4.3)$ boils down to a non-standard trace inequality

$$
\|\phi\|_{L^{q}(\Omega, d \sigma)} \leq C\|L \phi\|_{L^{1}(\Omega, d x)}, \quad \forall \phi \in C_{0}^{2}(\bar{\Omega}), \quad L \phi \geq 0 .
$$

It will be more convenient, as it often happens in the end-point case $p=1$, to replace (4.3) with a similar inequality for measures $v \in \mathcal{M}^{+}(\Omega)$ in place of $L^{1}(\Omega, d \sigma)$,

$$
\|G v\|_{L^{q}(\Omega, d \sigma)} \leq C\|v\|, \quad \forall v \in \mathcal{M}^{+}(\Omega),
$$

where $\|v\|=v(\Omega)$. Clearly, (4.5) $\Longrightarrow(4.3)$ if we set $d v=|f| d \sigma$. The converse is also true in many instances.

The following theorem demonstrates that a positive solution $u \in L^{q}(\Omega, \sigma)$ to (4.1) exists if and only if (4.5) holds. The same condition characterizes the existence of a positive supersolution $u \in L^{q}(\Omega, \sigma)$ such that

$$
u \geq G\left(u^{q} \sigma\right), \quad 0<u<+\infty \quad d \sigma-\text { a.e. in } \Omega .
$$

Theorem 4.1. Let $\sigma \in \mathcal{M}^{+}(\Omega)$ and $0<q<1$. Suppose $G$ is a lower semicontinuous, quasi-symmetric kernel which satisfies the weak maximum principle. Then the following statements are equivalent:

1) There exists a positive constant $C=C(\sigma, G)$ such that (4.5) holds.

2) There exists a supersolution $u \in L^{q}(\Omega, d \sigma)$ such that (4.6) holds.

3) There exists a solution $u \in L^{q}(\Omega, d \sigma)$ to (4.1).

This theorem is proved in [23] for more general non-negative kernels under the additional non-degeneracy assumption on the kernel $G$, which is in fact necessary for the existence of a positive solution. Non-degeneracy of $G$ is not needed for the existence of a non-trivial positive super-solution.

The next theorem gives some necessary and also close sufficient conditions for (4.5) to hold.

Theorem 4.2. Let $\sigma \in \mathcal{M}^{+}(\Omega)$, and $0<q<1$. Suppose $G$ is a quasi-symmetric, non-degenerate kernel which satisfies the weak maximum principle.

1) If (4.5) holds, then $G \sigma \in L^{\frac{q}{1-q}}(\Omega, \sigma)$.

2) If $G \sigma \in L^{\frac{q}{1-q}, 1}(\Omega, \sigma)$, then (4.5) holds.

Here $L^{s, 1}(\Omega, \sigma)$ is the corresponding Lorentz space (see [26]).

It is shown in [23] that, without the assumption that $G$ satisfies the weak maximum principle, the condition

$$
\int_{\Omega}(G \sigma)^{s} d \sigma<\infty
$$

with $s=\frac{q}{1-q}$ is necessary for the existence of a (super)solution $u \in L^{q}(\Omega, \sigma)$ to (4.1) only if $q \in\left(0, q_{0}\right]$, where

$$
q_{0}=\frac{\sqrt{5}-1}{2}=0.61 \ldots
$$

26 I.E. Verbitsky. Pointwise estimates of solutions and existence criteria for sublinear elliptic equations 
denotes the conjugate golden ratio. For general measures $\sigma$, the existence of a positive solution $u \in L^{q}(\Omega, \sigma)$ does not guarantee that (4.7) holds if $q \in\left(q_{0}, 1\right)$, or $s \neq \frac{q}{1-q}$ for all $q \in(0,1)$, even for symmetric positive kernels $G$.

Another characterization of the strong type inequality (4.5) can be deduced from Maurey's results [21]: it is equivalent to the existence of a nonnegative function $F \in L^{1}(\Omega, \sigma)$ which satisfies

$$
\sup _{y \in \Omega} \int_{\Omega} G(x, y) F(x)^{1-\frac{1}{q}} d \sigma(x)<+\infty .
$$

This is a dual reformulation of (4.5), which does not require $G$ to satisfy the weak maximum principle. In the discrete case where $\Omega$ consists of a finite number of points, it represents the duality of the two basic concave programming problems (see [1, Sec. 5.7]).

We also give in $[23 ; 25 ; 27]$ explicit characterizations of the weak type $(1, q)$-inequality

$$
\|G v\|_{L^{q, \infty}(\Omega, d \sigma)} \leq C\|v\|, \quad \text { for all } v \in \mathcal{M}^{+}(\Omega),
$$

for any $q>0$, in terms of energy estimates, as well as capacities.

In [23], we demonstrate how to remove the extra assumption imposed in Theorem 4.1 that a (super)solution $u \in L^{q}(\Omega, \sigma)$ globally. We prove the following theorem where we only assume that $u \in L_{\text {loc }}^{q}(\sigma)$, or equivalently, $0<u<+\infty d \sigma$-a.e., provided the kernel $G$ satisfies a weak form of the complete maximum principle, or alternatively if $G$ is a quasi-metric kernel (see, e.g., [11;18;23]).

The weak form (with constant $\mathfrak{b} \geq 1$ ) of the complete comparison principle, also called the domination principle, says the following:

$$
G \mu(x) \leq v(x) \text { on } \operatorname{supp}(\mu), G \mu<+\infty d \mu-\text { a.e. } \Longrightarrow G \mu(x) \leq \mathfrak{b} v(x) \text { on } \Omega,
$$

for any positive measure $\mu$ and positive superharmonic function $v$.

With a special function $m$ satisfying $0<m<+\infty d \sigma$-a.e., known as a modifier $[11 ; 18]$, we can modify the kernel $G$, so that the modified kernel

$$
K(x, y)=\frac{G(x, y)}{m(x) m(y)}, \quad x, y \in \Omega,
$$

satisfies the weak maximum principle (2.11). This makes it possible to apply Theorem 4.1 with $K$ in place of $G$, and consider $u \in L_{\text {loc }}^{q}(\Omega, \sigma)$. A typical modifier that works for general kernels $G$ which satisfy the complete maximum principle is given by

$$
m(x)=\min \left\{1, G\left(x, x_{0}\right)\right\}, \quad x \in \Omega,
$$

where $x_{0}$ is a fixed pole in $\Omega$ [19].

We say that $G$ is a quasimetric kernel with quasimetric constant $\mathrm{\kappa}>0$ provided $G$ is symmetric and $d(x, y):=\frac{1}{G(x, y)}$ satisfies the quasimetric triangle inequality with quasimetric constant $\kappa$, i.e.,

$$
d(x, y) \leq \kappa[d(x, z)+d(z, y)],
$$

for any $x, y, z \in \Omega$. Here $d(x, y): \Omega \times \Omega \rightarrow[0,+\infty)$.

The kernel $G$ is quasimetrically modifiable with constant $\mathrm{k}$ if there exists a modifier $m$ such that

$$
K(x, y)=\frac{G(x, y)}{m(x) m(y)}
$$

is a quasimetric kernel with quasimetric constant $\mathrm{k}$. 
Theorem 4.3. Let $\sigma \in \mathcal{M}^{+}(\Omega)$ and $0<q<1$. Suppose $G$ is a quasi-symmetric positive kernel, continuous in the extended sense on $\Omega \times \Omega$, which either (a) satisfies the complete maximum principle, or (b) is quasi-metrically modifiable. Then the following statements are equivalent:

1) There exists a positive constant $C$ such that the weighted norm inequality

$$
\|G v\|_{L^{q}(m d \sigma)} \leq C \int_{\Omega} m d v, \quad \text { for all } v \in \mathcal{M}^{+}(\Omega)
$$

holds, where the modifier $m(x)$ is given by (4.10) for some $x_{0} \in \Omega$.

2) There exists a positive (super) solution $u$ to (4.1) such that $u \in L_{\mathrm{loc}}^{q}(\Omega, \sigma)$ (or equivalently $0<u<+\infty$ do-a.e.)

Theorem 4.3 yields a characterization of the existence of weak solutions $u \in L_{\mathrm{loc}}^{q}(\Omega, \sigma)$ to the fractional Laplacian equation (1.5) in general domains $\Omega$ with positive Green's function $G$ for $0<\alpha \leq 2$, or the entire space $\mathbb{R}^{n}$, or balls, or half-spaces in $\mathbb{R}^{n}$, for $0<\alpha<n$ (quasi-metric kernels $G$ ) as discussed in the introduction. In the classical case $\alpha=2$, such solutions are the so-called very weak solutions for bounded $C^{2}$-domains $\Omega$ (see, e.g., [20]).

In the case $\Omega=\mathbb{R}^{n}$, an alternative criterion for the existence of weak solutions $u \in$ $\in L_{\text {loc }}^{q}\left(\mathbb{R}^{n}, \sigma\right)$ to the fractional Laplacian equation (3.2) based on localization of (4.5) is discussed in Sec. 3 above.

\section{Higher integrability of solutions}

Under the assumptions on the kernel $G$ imposed at the beginning of Sec. 4, let us consider conditions on $\sigma$ which ensure the existence of solutions $u \in L^{r}(\Omega, \sigma)$ with $r>0$ to the sublinear integral equation (4.1). In this case, a simple necessary and sufficient condition in terms of the energy (4.7) was obtained in [27]. In particular, these results were used in [24] to establish conditions for the existence of solutions $u \in L^{r}(\Omega, d x)$ (now with respect to Lebesgue measure) to the fractional Laplacian equation (1.5) in a general domain $\Omega$ with positive Green's function $G$, and also $W^{1, p}(\Omega)$ solutions to divergence form sublinear elliptic equations (1.3). These results are discussed in Sec. 6 below.

The following theorem (see [27]) characterizes the existence of solutions $u \in L^{r}(\Omega, \sigma)$ for $r>q$, along with related weighted norm inequalities

$$
\|G(f d \sigma)\|_{L^{r}(\Omega, \sigma)} \leq C\|f\|_{L^{p}(\Omega, \sigma)}, \quad \forall f \in L^{p}(\Omega, \sigma) .
$$

Theorem 5.1. Let $\sigma \in \mathcal{M}^{+}(\Omega)$. Suppose $G$ is a positive, lower semicontinuous, quasisymmetric kernel on $\Omega$ which satisfies the weak maximum principle.

(i) If $1<p<+\infty$ and $0<r<p$, then (5.1) holds if and only if

$$
\int_{\Omega}(G \sigma)^{\frac{p r}{p-r}} d \sigma<+\infty
$$

(ii) If $0<q<1$ and $q<r<\infty$, then there exists a positive (super) solution $u \in$ $\in L^{r}(\Omega, d \sigma)$ to (4.1) if and only if (5.1) holds with $p=\frac{r}{q}$, or equivalently by statement (i),

$$
\int_{\Omega}(G \sigma)^{\frac{r}{1-q}} d \sigma<+\infty
$$

28 I.E. Verbitsky. Pointwise estimates of solutions and existence criteria for sublinear elliptic equations 
Remark 5.2. We observe that the "if" parts of statements (i) and (ii) of Theorem 5.1 fail if $p=1$, and $r=q$, respectively. The "only if" parts hold for all $0<r<p$ in statement (i), and $r>0$ in statement (ii).

Remark 5.3. It is known that inequality (5.1) with $p=\frac{r}{q} \geq 1$ in the case $0<q<1$ yields the existence of a positive (super) solution $u \in L^{r}(\Omega, \sigma)$ for (4.1). This statement follows from a lemma due to Gagliardo [14], and does not require $G$ to be quasi-symmetric or to satisfy the weak maximum principle. However, the converse statement fails without the weak maximum principle (see [23] in the case $r=q$ ).

Remark 5.4. Without the assumption that $G$ satisfies the weak maximum principle, the "only if" parts of statement (i) (with $p=\frac{r}{q} \geq 1$ ) and statement (ii) (with $r \geq q$ ) of Theorem 5.1 hold only for $0<r \leq 1-q^{2}$; see [27]. The case $r=q$ when $0<q<q_{0}=\frac{\sqrt{5}-1}{2}$ is discussed above.

\section{Positive solutions in $W^{1, p}(\Omega)$ and $L^{r}(\Omega)$}

In this section, we present sharp conditions on the coefficient $\sigma$ which ensure the existence a positive weak solution $u \in W^{1, p}(\Omega)$ or $u \in L^{r}(\Omega, \sigma)$ to the sublinear elliptic Dirichlet problem

$$
\begin{cases}-\operatorname{div}(\mathcal{A} \nabla u)=\sigma u^{q}, & u>0 \text { in } \Omega, \\ u=0 & \text { on } \partial \Omega,\end{cases}
$$

where $0<q<1$, and $\sigma$ is a locally integrable function, or measure, in a domain $\Omega \subseteq \mathbb{R}^{n}$ with nonnegative Green's function $G$ associated with the uniformly elliptic operator $-\operatorname{div}(\mathcal{A} \nabla \cdot)$, for a symmetric matrix $\mathcal{A} \in L^{\infty}(\Omega)^{n \times n}$. Some of these results obtained in [24] hold for analogous fractional Laplacian equations (1.5) in domains $\Omega$ with positive Green's function.

More generally, for a lower semicontinuous kernel $G: \Omega \times \Omega \rightarrow(0,+\infty]$, where $\Omega \subseteq$ $\subseteq \mathbb{R}^{n}$, we denote by

$$
G(f d \sigma)(x)=\int_{\Omega} G(x, y) f(y) d \sigma(y), \quad x \in \Omega
$$

the corresponding integral operator, where $\sigma \in \mathcal{M}^{+}(\Omega)$, the class of positive locally finite Borel measures in $\Omega$. equation

We study positive solutions $u \in L^{r}(\Omega, d \sigma)$, as well as $u \in L^{r}(\Omega, d x)$, for the integral

$$
u=G\left(u^{q} d \sigma\right), \quad 0<u<+\infty \quad d \sigma-\text { a.e. in } \Omega,
$$

where $0<q<1$. If $G$ is Green's kernel associated with the Laplacian in a domain $\Omega \subseteq \mathbb{R}^{n}$, then (6.2) is equivalent to the sublinear elliptic boundary value problem (6.1) (see [23]).

As above, we assume that the kernel $G$ of the integral operator $G$ is lower semicontinuous, quasi-symmetric, and satisfies a weak maximum principle. In Theorem 6.4 below an additional assumption is imposed on $G$,

$$
G(x, y) \leq \frac{C}{|x-y|^{n-\alpha}}, \quad x, y \in \Omega
$$

for $0<\alpha<n$; here $C$ is a positive constant which does not depend on $x, y$. 
Such restrictions are satisfied by the Green kernel associated with many elliptic operators, including the fractional Laplacian $(-\Delta)^{\frac{\alpha}{2}}$ and $\mathcal{A}$-Laplacian (with $\alpha=2$ ) in a domain $\Omega \subseteq \mathbb{R}^{n}$.

The following theorems and corollaries are proved in [24].

Theorem 6.1. Let $\sigma \in \mathcal{M}^{+}(\Omega)$, where $\Omega \subseteq \mathbb{R}^{n}(n \geq 1)$ is a domain with a positive Green's function $G$ associated with $-\operatorname{div}(\mathcal{A} \nabla \cdot)$. Let $0<q<1$ and $\gamma>0$. Then there exists a positive solution $u$ to (6.1) such that

$$
\int_{\Omega}|\nabla u|^{2} u^{\gamma-1} d x<\infty
$$

if and only if

$$
\int_{\Omega}(G \sigma)^{\frac{\gamma+q}{1-q}} d \sigma<+\infty
$$

In particular, in the case $\gamma=1$, the condition

$$
\int_{\Omega}(G \sigma)^{\frac{1+q}{1-q}} d \sigma<+\infty
$$

is necessary and sufficient for the existence of a positive solution $u \in W_{0}^{1,2}(\Omega)$ to $(6.1)$.

Theorem 6.1 yields sufficient conditions for $u \in W_{0}^{1, p}(\Omega)$ when $\frac{n}{n-1}<p<2$. Here $W_{0}^{1, p}(\Omega)$ stands for the homogeneous Sobolev space defined as the closure of $C_{0}^{\infty}(\Omega)$ functions in the norm

$$
\|u\|_{W^{1, p}(\Omega)}=\left(\int_{\Omega}|\nabla u|^{p} d x\right)^{\frac{1}{p}}
$$

Letting $p=\frac{n(1+\gamma)}{n-1+\gamma}$ with $0<\gamma<1$ in Theorem 6.1 , we obtain the following corollary.

Corollary 6.2. Under the assumptions of Theorem 6.1, suppose that $\frac{n}{n-1}<p<2$ and $n \geq 3$. If

$$
\int_{\Omega}(G \sigma)^{\frac{p(n-2)}{(1-q)(n-p)}-1} d \sigma<+\infty
$$

then there exists a positive solution $u \in W_{0}^{1, p}(\Omega)$ to $(6.1)$.

Corollary 6.3. Under the assumptions of Theorem 6.1, suppose $\sigma \in L^{s}(\Omega)$, where $\frac{n}{n-q(n-2)}<$ $<s \leq \frac{2 n}{n+2-q(n-2)}$. Then there exists a positive solution $u \in W_{0}^{1, p}(\Omega) \cap L^{r}(\Omega)$ to (6.1) with $p=\frac{n(1-q) s}{n-s(1+q)}$ and $r=\frac{n(1-q) s}{n-2 s}$.

In the following theorem we give conditions for the existence of positive solutions $u \in L^{r}(\Omega, d x)$ for more general kernels $G$.

Theorem 6.4. Let $\Omega \subseteq \mathbb{R}^{n}(n \geq 1)$, and $\sigma \in \mathcal{M}^{+}(\Omega)$. Let $0<q<1$. Suppose $G$ is a positive, lower semicontinuous kernel on $\Omega \times \Omega$ which is quasi-symmetric and satisfies the weak maximum principle.

(i) If there exists a solution $u \in L^{r}(\Omega, d x), r>0$, to (6.2), then

$$
\int_{\Omega}(G \sigma)^{\frac{r}{1-q}} d x<+\infty .
$$

(ii) Suppose additionally that $G$ satisfies condition (6.3) for some $0<\alpha<n$. If $\frac{n}{n-\alpha}<r<\infty$ and

$$
\int_{\Omega}(G \sigma)^{\frac{r(n-\alpha)}{(1-q) n}-1} d \sigma<+\infty
$$

30 I.E. Verbitsky. Pointwise estimates of solutions and existence criteria for sublinear elliptic equations 
then there exists a positive solution $u \in L^{r}(\Omega, d x)$ to (6.2).

Corollary 6.5. Under the assumptions of Theorem 6.4, suppose that $\sigma \in L^{s}(\Omega, d x)$, where $\frac{n}{n-q(n-\alpha)}<s<\frac{n}{\alpha}$. Then there exists a positive solution $u \in L^{r}(\Omega, d x)$ to (6.2), where $r=\frac{n(1-q) s}{n-\alpha s}$.

Corollaries 6.3 and 6.5 for bounded domains $\Omega$ were obtained earlier by Boccardo and Orsina [4]. We observe that the restrictions on $s$ in these corollaries, and consequently on the exponents $\gamma, p$ and $r$ in Theorem 6.1, Theorem 6.4, and Corollary 6.2, are sharp.

There are also weak-type analogues of these results for Marcinkiewicz spaces $L^{r, \infty}$ as in [5], based on the corresponding weak-type weighted norm inequalities (see $[23 ; 25 ; 27]$ ).

The proof of Theorem 6.1 in [24] makes use of the weighted norm inequality

$$
\|G(f d \sigma)\|_{L^{r}(\Omega, d \sigma)} \leq C\|f\|_{L^{s}(\Omega, d \sigma)}, \quad \forall f \in L^{s}(\Omega, d \sigma),
$$

for integral operators $G$ in the case $1<r<s<+\infty$ considered in Sec. 3.

The proof of Theorem 6.4 uses both (6.10) and a similar weighted norm inequality with Lebesgue measure on the left-hand side,

$$
\|G(f d \sigma)\|_{L^{r}(\Omega, d x)} \leq C\|f\|_{L^{s}(\Omega, d \sigma)}, \quad \forall f \in L^{s}(\Omega, d \sigma),
$$

for $1<r<\infty, 1<s<+\infty$. Such inequalities in the equivalent dual form

$$
\|G f\|_{L^{s^{\prime}}(\Omega, d \sigma)} \leq C\|f\|_{L^{r^{\prime}(\Omega, d x)}}, \quad \forall f \in L^{r^{\prime}}(\Omega, d x),
$$

were characterized in the special case where $\Omega=\mathbb{R}^{n}$ and $G=(-\Delta)^{-\frac{\alpha}{2}}$ is the Riesz potential of order $\alpha \in(0, n)$, by Maz'ya for $r \geq s \geq 1$, and Maz'ya and Netrusov for $1<r<s$, in terms of Sobolev capacities, and later by Cascante, Ortega and the author in terms of Wolff potentials (see $[7 ; 22]$ ).

In [24], we employ an alternative characterization in terms of Green's potentials $G \sigma$ as in Theorem 5.1 above, which is sufficient for both (6.10) and (6.11) simultaneously.

\section{REFERENCES}

1. Adams D.R., Hedberg L.I. Function Spaces and Potential Theory. Berlin; Heidelberg; New York, Springer-Verlag, 1996. 368 p.

2. Ancona A. Some results and examples about the behaviour of harmonic functions and Green's functions with respect to second order elliptic operators. Nagoya Math. J., 2002, vol. 165, pp. 123-158.

3. Berge C., Ghouila-Houri A. Programming, Games and Transportation Networks. London, Methuen, 1965. 248 p.

4. Boccardo L., Orsina L. Sublinear elliptic equations in $L^{s}$. Houston Math. J., 1994, vol. 20, pp. 99-114.

5. Boccardo L., Orsina L. Sublinear elliptic equations with singular potentials. Adv. Nonlin. Studies, 2012, vol. 12, pp. 187-198.

6. Brezis H., Kamin S. Sublinear elliptic equation on $\mathbb{R}^{n}$. Manuscr. Math., 1992, vol. 74, pp. $87-106$.

7. Cascante C., Ortega J.M., Verbitsky I.E. On $L^{p}-L^{q}$ trace inequalities. J. London Math. Soc., (2006), vol. 74, pp. 497-511.

8. Cao D.T., Verbitsky I.E. Nonlinear elliptic equations and intrinsic potentials of Wolff type. J. Funct. Analysis, 2017, vol. 272, pp. 112-165. DOI: http://dx.doi.org/10.1016/j.jfa.2016.10.010. 
9. Cao D.T., Verbitsky I.E. Pointwise estimates of Brezis-Kamin type for solutions of sublinear elliptic equations. Nonlin. Analysis Ser. A: Theory, Methods \& Appl., 2016, vol. 146, pp. 1-19.

10. Dat C.T., Verbitsky I.E. Finite energy solutions of quasilinear elliptic equations with sub-natural growth terms. Calc. Var. PDE, 2015, vol. 52, pp. 529-546.

11. Frazier M., Nazarov F., Verbitsky I. Global estimates for kernels of Neumann series and Green's functions. J. London Math. Soc., 2014, vol. 90, pp. 903-918.

12. Fuglede B. On the theory of potentials in locally compact spaces. Acta Math., 1960, vol. 103, pp. 139-215. $482 \mathrm{p}$

13. Grigor'yan A. Heat Kernel and Analysis on Manifolds. Boston, AMS, 2009.

14. Grigor'yan A. Heat kernels on weighted manifolds and applications. Contemp. Math., 2006, vol. 398, pp. 93-191.

15. Grigor'yan A., Hansen W. Lower estimates for a perturbed Green function. J. Anal. Math., 2008, vol. 104, pp. 25-58.

16. Grigor'yan A., Verbitsky I.E. Pointwise estimates of solutions to nonlinear equations for nonlocal operators, preprint, 2017.

17. Grigor'yan A., Verbitsky I.E. Pointwise estimates of solutions to semilinear elliptic equations and inequalities. J. d'Analyse Math. (to appear). URL: https://arxiv.org/abs/1511.03188.

18. Hansen W. Uniform boundary Harnack principle and generalized triangle property. J. Funct. Analysis, 2005, vol. 226, pp. 452-484.

19. Hansen W., Netuka I. On the Picard principle for $\Delta+\mu$. Math. Z., 2012, vol. 270, pp. 783-807.

20. Marcus M., Véron L. Nonlinear Second Order Elliptic Equations Involving Measures. Berlin; Boston, Walter de Gruyter, 2014. 248 p.

21. Maurey B. Théorèmes de factorisation pour les opérateurs linéaires à valeurs dans les espaces $L^{p}$. Paris, Soc. Math. France, 1974. 384 p.

22. Maz'ya V. Sobolev Spaces, with Applications to Elliptic Partial Differential Equations. Berlin, Springer, 2011. 866 p.

23. Quinn S., Verbitsky I.E. A sublinear version of Schur's lemma and elliptic PDE, preprint. URL: https://arxiv.org/abs/1702.02682.

24. Quinn S., Verbitsky I.E. Solutions to sublinear elliptic equations in $W^{1, p}(\Omega)$ and $L^{r}(\Omega)$ spaces, preprint, 2017.

25. Quinn S., Verbitsky I.E. Weighted norm inequalities of $(1, q)$-type for integral and fractional maximal operators. Harmonic Analysis, Partial Differential Equations and Applications, in Honor of Richard L. Wheeden, Ser. Applied and Numerical Harmonic Analysis. Basel, Birkhäuser, 2017, pp. 217-238.

26. Stein E., Weiss G. Introduction to Fourier Analysis on Euclidean Spaces. Princeton, N.J., Princeton Univ. Press, 1971. 297 p.

27. Verbitsky I.E. Sublinear equations and Schur's test for integral operators, 50 Years with Hardy Spaces, a Tribute to Victor Havin, Ser. Operator Theory: Adv. Appl., vol. 261, Basel, Birkhäuser, 2017 (to appear). 


\section{ПОТОЧЕЧНЫЕ ОЦЕНКИ И КРИТЕРИЙ СУЩЕСТВОВАНИЯ РЕШЕНИЙ СУБЛИНЕЙНЫХ ЭЛЛИПТИЧЕСКИХ УРАВНЕНИЙ}

\section{Игорь Е. Вербицкий}

Профессор, Факультет математики, University of Missouri verbitskyi@missouri.edu Columbia, MO 65211, USA

Аннотация. В работе представлен обзор последних результатов о положительных решениях эллиптических уравнений типа $-L u+V u^{q}=f$, где $L-$ эллиптический оператор в дивергентной форме, $0<q<1, f \geq 0$ и $V-$ функция, которая может изменять знак, в области $\Omega \subseteq \mathbb{R}^{n}$ или на весовом римановом многообразии с положительной функцией Грина $G$. Обсуждаются вопросы существования решений, глобальные нижние и верхние поточечные оценки классических и слабых решений $u$, а также условия, обеспечивающие $u \in L^{r}(\Omega)$ или $u \in W^{1, p}(\Omega)$.

Некоторые из этих результатов применимы к однородным сублинейным интегральным уравнениям $u=G\left(u^{q} d \sigma\right)$ in $\Omega$, где $0<q<1$, а $\sigma=-V-$ положительная локально конечная борелевская мера в $\Omega$. Здесь $G(f d \sigma)(x)=$ $=\int_{\Omega} G(x, y), f(y) d \sigma(y)$ - интегральный оператор с положительным (квази) симметричным ядром $G$ на $\Omega \times \Omega$, который удовлетворяет слабому принципу максимума. Результаты распространяются на положительные, возможно сингулярные, решения сублинейных уравнений, содержащих дробный лапласиан

$$
(-\Delta)^{\frac{\alpha}{2}} u=\sigma u^{q}, \quad u \geq 0 \quad \text { в } \Omega,
$$

где $0<q<1,0<\alpha<n$ и $u=0$ в $\Omega^{c}$ и на бесконечности в областях $\Omega \subseteq \mathbb{R}^{n}$ с положительной функцией Грина $G$.

Ключевые слова: сублинейные эллиптические уравнения, функция Грина, слабый принцип максимума, дробный лапласиан. 